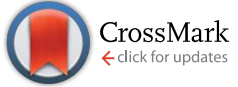

Cite this: DOI: $10.1039 / \mathrm{c} 4 \mathrm{sm} 01356 \mathrm{e}$

Received 23rd June 2014 Accepted 25th July 2014

DOI: $10.1039 / \mathrm{c} 4 \mathrm{sm} 01356 \mathrm{e}$

www.rsc.org/softmatter

\title{
Mesomorphism of a new series of catanionic 4-(1-pentylheptyl)benzenesulfonates $\uparrow$
}

\author{
Vlasta Tomašić, ${ }^{\star a}$ Tea Mihelj, ${ }^{a}$ Ruibin Zhang, ${ }^{b}$ Feng Liư and Goran Ungar ${ }^{\text {bd }}$
}

The effect of cationic chemical structure on the mesophase behavior, and self-assembly of catanionic compounds is studied through a systematic structure variation of six cationic surfactants with anionic sodium 4-(1-pentylheptyl)benzenesulfonate surfactant. A series of cationic surfactants are selected with an increasing number of $n$-dodecyl chains on the same ammonium headgroup (from one to three), and with an increasing number of ammonium headgroups (from two to four). Thermal and phase behavior of the synthesized products were examined by thermogravimetry, polarizing microscopy, differential scanning calorimetry, powder and grazing incidence X-ray diffraction, and molecular simulation. The compounds are complexes of high thermal stability. Most of the thermotropic mesophases are smectic, although the hexagonal mesophase is also observed. Introduction of the third dodecyl chain into the cationic part leads to pronounced lower melting temperatures and eradicates mesomorphism, while the addition of a fourth ammonium headgroup leads to a catanionic benzenesulfonate that decomposes simultaneously under melting. Melting and crystallization temperatures show nonlinear dependence on the total number of alkyl chains, and on the number of headgroups in the molecule. Molecular dynamics simulation of the smectic phase points to separated hydrophilic and hydrophobic layers, favoring headto-head packing of antiparallel molecules. On the other hand, there are two ion pairs in the column cross-section of the hexagonal LC phase of didodecyldimethylammonium-4-(1-pentylheptyl)benzenesulfonate.

\section{Introduction}

Surfactant molecules possess the ability to self-organize in the process by which molecules spontaneously and reversibly, without external stimuli, adopt a defined intermolecular ordered arrangement. The strong tendency of two oppositely charged surfactants to mix results in a range of self-associated structures governed by electrostatic interactions, geometrical packing, repulsive hydration forces, the attractive hydrophobic interactions and entropic factors. ${ }^{1-4}$ Most mixed cationicanionic surfactant systems, precipitate at equimolar concentrations, making uncharged complexes or ion-pair amphiphiles that contain only parent surfactants, without inorganic

${ }^{a}$ Department of Physical Chemistry, Ruđer Bošković Institute, Laboratory for synthesis and processes of self-assembling of organic molecules, Bijenička c. 54, P.O. Box 180, HR-10002 Zagreb, Croatia. E-mail: Vlasta.Tomasic@irb.hr; Fax: +38 514680245; Tel: +38514571211

${ }^{b}$ Department of Material Science and Engineering, University of Sheffield, Sheffield S1 3JD, UK

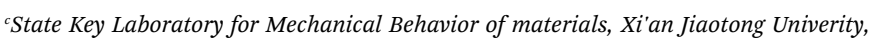
$X i^{\prime} a n, 710049$, China

${ }^{d}$ Department of Physics, Zhejiang Sci-Tech Univerity, Xiasha College Park, Hangzhou, 310018 China

$\uparrow$ Electronic supplementary information (ESI) available. See DOI: $10.1039 / \mathrm{c} 4 \mathrm{sm} 01356 \mathrm{e}$ counterions. Although considerable efforts have been made to design and synthesize new types of catanionic materials, most of them have been focused on their aqueous solutions, precipitate-solubility boundaries and characterization of their physico-chemical behavior. These were mostly examinations of micellar and vesicular self-assembly, ${ }^{\mathbf{1 , 2 , 5 - 1 2}}$ or of the formation and structure of the lamellar phase comparable with mechanisms in biological membranes, ${ }^{13}$ while at the same time studies of their bulk solid state are relatively scarce.,.$^{3,4,14-24}$ The structure of catanionic aggregates can be fine-tuned by tailoring the nature of the components, owing to the interplay between electrostatic effects, surfactants' molecular size and geometry, and hydrogen bonding network. Design of different complexes and tailored functionalized assemblies opens opportunities for potential applications in commercial products; from transport agents in drug and gene delivery ${ }^{5}$ and template materials, to sensors and semiconductors, ${ }^{6}$ information storage and nanotechnology. ${ }^{5,25}$ Liquid crystals with different structures derived from surfactants, lamellar, columnar, and cubic, provide a facile strategy to construct defined functional materials. Surfactant compounds also play an important role in life sciences. The formation of liquid crystal phases, e.g. closed bilayer aggregates (vesicles) or plain bilayers (lamellae), makes them reliable models that imitate self-association of lipids into membranes and living tissues. 
The formation of liquid crystals by catanionics is not restricted to lyotropic systems, but is also an interesting property in terms of their thermal behavior. Solid catanionics exhibit complex polymorphic and mesomorphic behavior during thermal treatment..$^{3,4,14,17-22,26}$ The occurrence of mesophases depends on the geometric shape of the units, and the distribution of polar groups in the molecule. As the difference in pendant chain lengths between cation and anion increases, a poorer hydrophobic match in the bilayer is attained. ${ }^{4,15,16}$ The common feature of most catanionics is the layer-like arrangement in crystal phases, mostly obtained as the crystal smectic (Sm) phase. Alkyl benzenesulfonates are a very important class of anionic surfactants, used in domestic and industrial applications and often providing excellent cleaning, wetting, foaming, dispersing, and emulsifying abilities. They are usually used as sodium salts. They have found broad applications in microemulsion production, ${ }^{27}$ synthetic, ${ }^{28-33}$ analytical, ${ }^{32,34-36}$ and environmental ${ }^{37-39}$ chemistry. Commercial linear $n$-alkyl benzenesulfonates are usually complex mixtures of homologues of different chain lengths and positional isomers. ${ }^{12,40}$ Relatively few studies on pure homologues and isomers are available..$^{22,40,41}$ An example is our previous study of 4-(1-pentylheptyl)benzenesulfonate. $^{22}$ Sulfonate based catanionic molecules or metal complexes often have some kind of layer-like arrangement in the crystals. $^{\mathbf{1 7 , 1 8 , 4 2 - 4 4}}$ Calixarenes based on sulfonates show typical bilayer structures with calixarene molecules held together by $\pi-\pi$ interactions forming hydrophobic layers and sulfonate groups covering the hydrophilic surfaces of the bilayer. ${ }^{44}$ The crystal smectic phase of guanidinium alkanesulfonates consists of alternating ionic and alkyl sheets arranged on a hexagonal lattice. ${ }^{18}$ Alkylammonium benzenesulfonates, naphthalenesulfonates and related salts form SmA mesophases. ${ }^{\text {20-22 }}$ Most known catanionic compounds based on alkylammonium are bilayered structures, ${ }^{3,4}$ but also some of them, such as alkylammonium-AOT complexes, form hexagonal columnar phases. ${ }^{\mathbf{1 4}}$ Hexadecyl and octadecyl derivatives of alkylammonium poly(vinylsulfonate)s, ${ }^{17}$ like hexadecylpyridinium and hexadecyltrimethylammonium 4-octylbenzenesulfonate, ${ }^{19}$ exhibit low temperature $\mathrm{SmB}$ and high temperature SmA phases, while dodecyl and tetradecyl derivatives of $N, N$ dimethyl- $N$-cyanopropyl- $n$-alkylammonium poly(vinylsulfonate) s form cubic phases that consist of warped molecular bilayers. ${ }^{17}$

In the present work six catanionic 4-(1pentylheptyl)benzenesulfonates were synthesized, differing in the cationic part of the molecule. The chosen cationic parts are quaternary ammonium salts, previously studied in terms of their thermal behavior: dodecyltrimethylammonium bromide ${ }^{\mathbf{4 5}}$ didodecyldimethylammonium bromide, ${ }^{\mathbf{4 6}}$ tridodecylmethylammonium chloride ${ }^{47}$ and oligomeric bromides based on dodecyltrimethylammonium bromide. ${ }^{48}$ The number of dodecyl chains on the same ammonium headgroup and the number of quaternary ammonium headgroups are varied in this study. Thermal stability, the thermotropic phase sequence, temperatures and heats of transitions, and phase structures were examined using thermogravimetry, optical microscopy, differential scanning calorimetry and grazing-incidence X-ray diffraction. The main goal of the study is to understand the relationship between chemical structure, molecular arrangement and thermal behavior, especially mesophase formation.

\section{Experimental}

\subsection{Materials}

Dodecyltrimethylammonium bromide, DTAB (Fluka, >99.0\% AT), didodecyldimethylammonium bromide, DDAB (Acros Organics, $>99.0 \%$ AT), and tridodecylmethylammonium chloride TDMAC (Fluka, $\geq 97.0 \%$ AT) were used without further purification for the preparation of solid salts. Oligomeric surfactant bromides with the same spacer group separating the monomer units were synthesized: ${ }^{48} \quad$ bis( $N, N$-dimethyl- $n$-dodecyl)ethylene-1,2-diammonium dibromide, (12-2-12), 2Br; $N$-bis $\left[2-\left(N^{\prime}, N^{\prime}\right.\right.$-dimethyl- $n$ dodecylammonio)ethylene]- $n$-dodecyl- $N$-methyl-1,2-diammonium tribromide, (12-2-12-2-12), 3Br.; $N, N^{\prime}$-bis $\left[2^{\prime}-\left(N^{\prime \prime}, N^{\prime \prime}\right.\right.$-dimethyl- $n$ dodecylammonio)ethylene]-di- $n$-dodecyl- $N, N^{\prime}$-dimethyl-ethylene1,2-diammonium tetrabromide, (12-2-12-2-12-2-12), $4 \mathrm{Br}$. Sodium 4-(1-pentylheptyl)benzenesulfonate (analytically pure) was a present courtesy of the Henkel Co., Germany; it was further purified by multiple recrystallization from ethanolwater to remove excess of inorganic electrolyte, and its purity was established previously. ${ }^{22}$ Water used in this study was purified using a Millipore MilliQ system.

\subsection{Preparation of surfactant molecular complexes}

The examined complexes are shown in Scheme 1: dodecyltrimethylammonium-4-(1-pentylheptyl)benzenesulfonate (compound 1); didodecyldimethylammonium-4-(1-pentylheptyl)benzenesulfonate (2); tridodecylmethylammonium-4-(1pentylheptyl)benzenesulfonate (3); bis( $N, N$-dimethyl- $n$-dodecyl)ethylene-1,2-diammonium di-(4-(1-pentylheptyl)benzenesulfonate) (4); $N$-bis $\left[2-\left(N^{\prime}, N^{\prime}\right.\right.$-dimethyl- $n$ - dodecylammonio)ethylene $]$ $n$-dodecyl- $N$-methyl-1,2-diammonium tri-(4-(1-pentylheptyl)benzenesulfonate) (5); and $N, N^{\prime}$-bis $\left[2^{\prime}-\left(N^{\prime \prime}, N^{\prime \prime}\right.\right.$-dimethyl- $n$-dodecylammonio)ethylene]-di- $n$-dodecyl- $N, N^{\prime}$-dimethyl ethylene-1,2diammonium tetra-(4-(1-pentylheptyl)benzenesulfonate) (6). Surfactant molecular complexes were prepared from equimolar concentrations of appropriate surfactant aqueous solutions by mixing with a magnetic stirrer for 45 minutes at elevated temperature, and equilibrated undisturbed for at least one week. Exceptionally, compounds $\mathbf{3}$ and $\mathbf{4}$ were prepared in ethanol. The precipitated compounds were filtered through Millipore filter type HA with $0.45 \mu \mathrm{m}$ pore size, washed with cold water to remove coprecipitated salts $(\mathrm{NaBr}$ or $\mathrm{NaCl}$ ), with diethylether to remove possibly coprecipitated cationic or anionic surfactants, and then dried under vacuum. Such waxy and semi-transparent samples of brightly yellow to brown color were stored in a dessicator, protected from light and moisture before further analysis.

\subsection{Measurements}

Identification and purity evaluation of catanionic compounds were performed by elemental analysis (Perkin-Elmer Analyzer PE 2400 Series 2). Elemental analyses (mass percentages) 


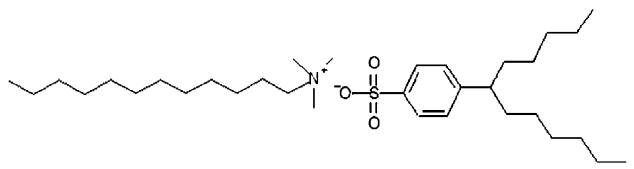

1

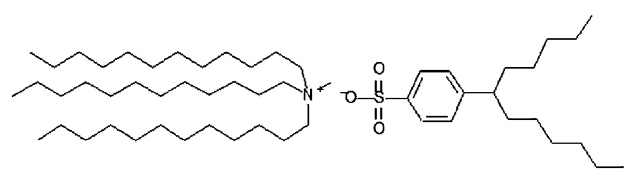

3

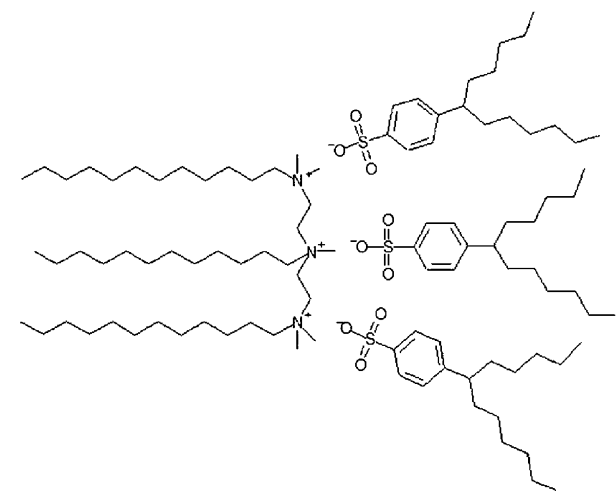

5

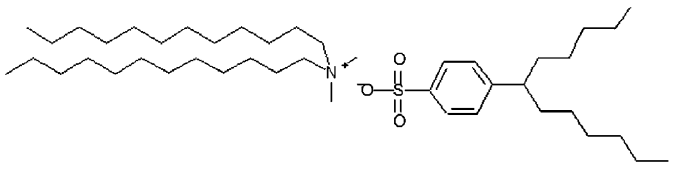

2

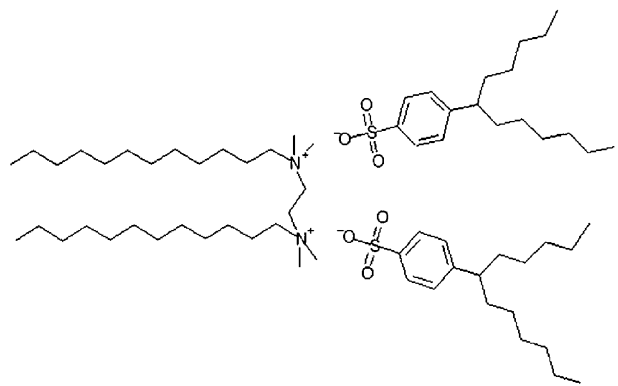

4

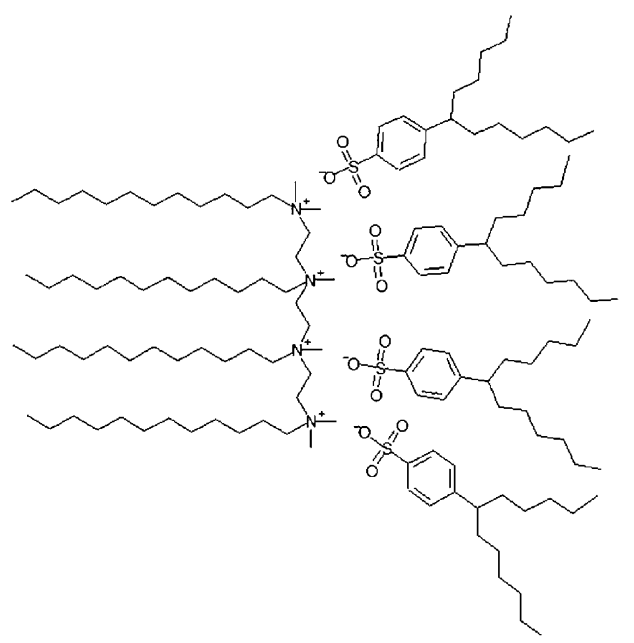

6

Scheme 1 The scheme of the examined catanionic 4-(1-pentylheptyl)benzenesulfonates: an increasing number of alkyl chains on the same ammonium headgroup (compounds 1-3); and an increasing number of ammonium headgroups (compounds 4-6).

confirmed that the complexes were $1: 1$ (charge ratio) adducts of high purity.

Compound 1, $\mathrm{C}_{35} \mathrm{H}_{59} \mathrm{NO}_{3} \mathrm{~S}, M_{\mathrm{w}}=$ 553.95. Found: C, 73.24; $\mathrm{H}$, 10.33; N, 2.47; requires C, 73.25; H, 10.36; N, 2.44\%. Compound $2, \mathrm{C}_{44} \mathrm{H}_{85} \mathrm{NO}_{3} \mathrm{~S}, M_{\mathrm{w}}=708.23$. Found: C, 74.63; H, 12.07; N, 1.94; requires $\mathrm{C}, 74.62 ; \mathrm{H}, \quad 12.10 ; \mathrm{N}, \quad 1.98 \%$. Compound $3, \mathrm{C}_{55} \mathrm{H}_{107} \mathrm{NO}_{3} \mathrm{~S}, M_{\mathrm{w}}=862.53$. Found: $\mathrm{C}, 76.63 ; \mathrm{H}, 12.54 ; \mathrm{N}, 1.64$; requires $\mathrm{C}, 76.59 ; \mathrm{H}, 12.50 ; \mathrm{N}, 1.62 \%$. Compound 4, $\mathrm{C}_{66} \mathrm{H}_{124} \mathrm{~N}_{2} \mathrm{O}_{6} \mathrm{~S}_{2}, M_{\mathrm{w}}=1105.86$. Found: C, 71.67; H, 11.34; $\mathrm{N}, 2.56$; requires $\mathrm{C}, 71.68 ; \mathrm{H}, 11.30 ; \mathrm{N}, 2.53 \%$. Compound 5, $\mathrm{C}_{99} \mathrm{H}_{185} \mathrm{~N}_{3} \mathrm{O}_{9} \mathrm{~S}_{3}, M_{\mathrm{w}}=1657.77$. Found: C, 71.67; H, 11.34; $\mathrm{N}, 2.56$; requires $\mathrm{C}, 71.73 ; \mathrm{H}, 11.25 ; \mathrm{N}, 2.53 \%$. Compound 6, $\mathrm{C}_{132} \mathrm{H}_{247} \mathrm{~N}_{4} \mathrm{O}_{12} \mathrm{~S}_{4}, M_{\mathrm{w}}=2209.71$. Found: $\mathrm{C}, 71.77 ; \mathrm{H}, 11.31 ; \mathrm{N}$, 2.56; requires C, 71.75; $\mathrm{H}, 11.27$; N, 2.54\%.

Thermogravimetric analysis, TGA, was performed from room temperature (RT) to $287{ }^{\circ} \mathrm{C}$, with a Mettler TA 4000 System. It gave the following decomposition temperatures: $t_{\mathrm{d}} /{ }^{\circ} \mathrm{C}=257$ (1), 240 (2), 220 (3), 270 (4), 237 (5), and 283 (6).
Thermal properties were examined by differential scanning calorimetry, DSC, using a Perkin Elmer Pyris Diamond DSC calorimeter in a $\mathrm{N}_{2}$ atmosphere, equipped with a model Perkin Elmer $2 \mathrm{P}$ intra-cooler. Heating runs were recorded from RT to different $t /{ }^{\circ} \mathrm{C}: 227$ (1), 187 (2), 27 (3), 267 (4), 202 (5), and 287 (6), followed by cooling runs to RT at a rate of $5 \mathrm{~K} \mathrm{~min}^{-1}$. The transition enthalpy, $\Delta H / \mathrm{kJ} \mathrm{mol}^{-1}$, was determined from the peak area of the DSC thermogram, and the corresponding entropy change, $\Delta S / \mathrm{J} \mathrm{mol}^{-1} \mathrm{~K}^{-1}$, was calculated using the peak transition temperatures. All results are mean values of three independent measurements with the standard deviation being of the order of $5 \%$. Temperature and enthalpy calibrations were performed using high purity standard (indium). Due to different periods of catanionic surfactant recovery to the original state after heating, ${ }^{3,4,15,16}$ only data obtained on first scans are reported. Textures were examined with a Leica DMLS and an Olympus BX50 polarized optical microscope, equipped, respectively, with a Sony SSC-DC58AP and a Peltier-cooled Coolsnap (ImagePro) digital camera. Linkam LNP (THMS 600) 
hot stages were used, with liquid nitrogen cooling where required. Video sequences were recorded in a slow-scan mode using ImagePro camera control software. The orderly arrangement of molecules in some crystals results in different resonant frequencies for different orientations of the electric vector relative to the crystalline axes, which results in different refractive indices for different polarizations. We used birefringence to modify the polarization state of light with optical components called birefringent wave plates or retardation plates. We say that the wave is polarized along the fast axis, since its phase velocity will be a maximum. A plane-polarized wave with its plane rotated $90^{\circ}$ will propagate with the maximum index of refraction and minimum phase velocity. We say it is polarized along the slow axis.

Powder X-ray diffraction (PXRD) experiments were done at room and high temperatures on a theta-theta diffractometer (Ultima III, Rigaku, Japan) equipped with a sample oven and a scintillation counter. The step size was $0.02^{\circ}$ and the scan rate was $0.5^{\circ}$ per minute. Data were analyzed in Jade 9 (MDI, Inc., CA). A number of diffraction lines, having a detectable intensity at room temperature, were identified as multiple orders of diffraction from the crystal planes corresponding to the basic lamellar thickness (the long spacing), and these diffraction lines were given Miller indices $00 l$. The interlayer spacings, $d_{00 l}$, were calculated according to Bragg's law. The intensity was calibrated through the use of a calibration sample, that is silicium, the scattering of which is known before. The experimental error for PXRD was estimated to be no higher than $0.1 \%$.

Low temperature measurements were made on a homemade small angle X-ray scattering (SAXS) instrument with an Oxford Cryostream cooling system. A home-made linear position-sensitive detector was used for detection. High resolution powder SAXS experiments were carried out at beamline I22 of a Diamond Light Source. Grazing-induce SAXS (GISAXS) experiments on a thin film sample on a Si substrate were carried out at XMasS (BM28) beamline at ESRF. In both later cases a MAR195 CCD area detector was used. Temperature control was provided in these cases by a Linkam hot stage and a home built Eurotherm-controlled sample platform, respectively. Molecular model building and molecular dynamics simulations were performed in Material Studio (Accelrys Inc.), using the ForcitePlus module and Universal force field. Constant volume unit cell dimensions were fixed based on experimental data. The application of these corrections can bring the detected intensity accuracy to $1-2 \%$.

\section{Results and discussion}

The compounds examined here (Scheme 1) are molecular ionpair complexes, containing the same anionic part 4-(1-pentylheptyl)benzenesulfonate, but differing in the cationic part. The compounds can be divided into two main groups: those with an increasing number of alkyl chains attached to the same headgroup (1-3), and those, referred to as oligomers, with an increasing number of headgroups, and by implication, alkyl chains in the molecule (4-6).

Table 1 presents the transition temperatures and the related enthalpy and entropy changes. The general trend is for the more complex chemical structures to exhibit a larger number of polymorphs. Considering the temperatures, a direct systematic trend cannot be observed within the studied compounds 1-6. Comparison of dodecylammonium-4-(1-pentylheptyl)-

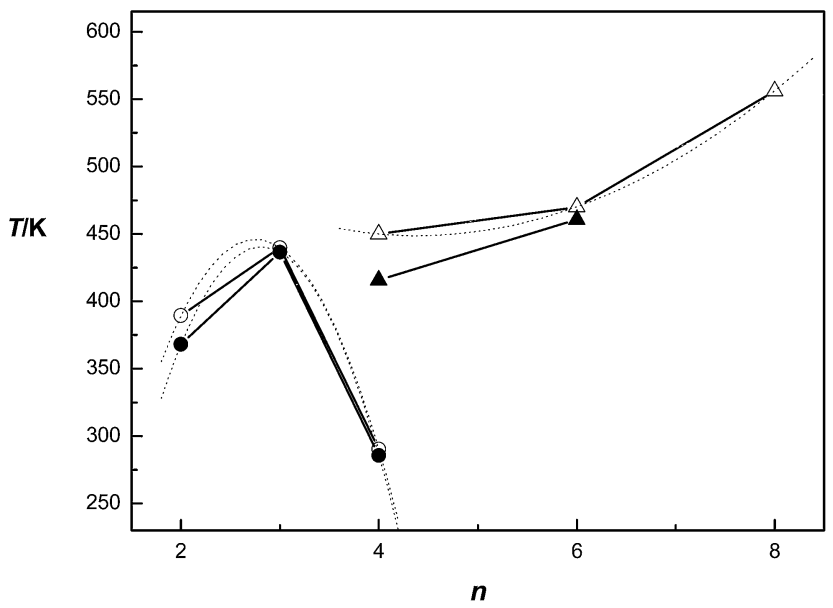

Fig. 1 The melting $(O, \triangle)$ and the crystallization $(\boldsymbol{O}, \boldsymbol{\Delta})$ temperatures, $T / K$, as a function of the number of dodecyl chains, $n$, for multitailed $\left(n_{1}=2-4\right.$, compounds $\left.1-3\right)$ and multi-charged $\left(n_{2}=4-8\right.$, compounds 4-6) 4-(1-pentylheptyl)benzenesulfonate catanionic catanionics.

Table 1 Transition temperatures, $T / K$, enthalpy changes, $\Delta H / \mathrm{KJ} \mathrm{mol}^{-1}$, and entropy changes, $\Delta S / \mathrm{J} \mathrm{mol}^{-1} \mathrm{~K}^{-1}$, of the examined catanionics. Letter $n$ denotes the total number of $n$-dodecyl chains in the multi-chained, $n_{1}$; or in the multi-headed compounds, $n_{2}$

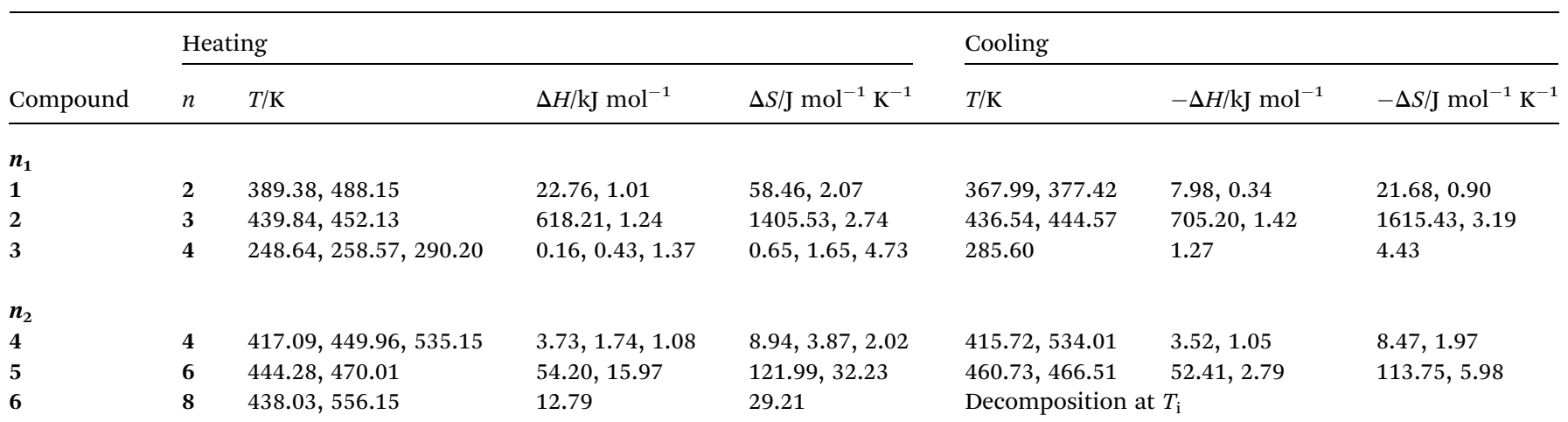


benzenesulfonate ${ }^{22}$ and 1 shows higher melting and crystallization temperatures of the methylated compound. Compound 2 shows higher melting $\left(220{ }^{\circ} \mathrm{C}\right)$, clearing and crystallization

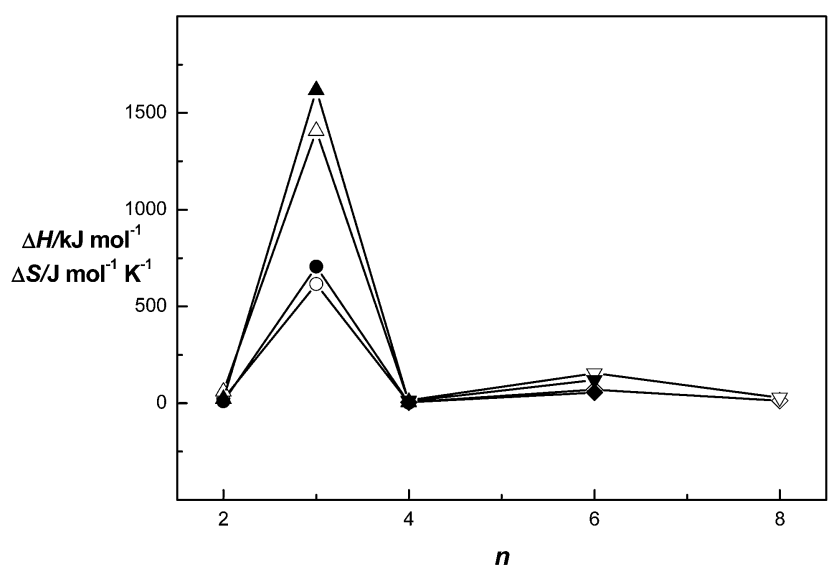

Fig. 2 Absolute values of changes in total enthalpy, $\Delta H / \mathrm{kJ}$ $\mathrm{mol}^{-1}(\bigcirc, \diamond)$, and entropy changes, $\Delta S / \mathrm{J} \mathrm{mol}^{-1} \mathrm{~K}^{-1}(\Delta, \nabla)$, during heating (open symbols) and cooling (solid symbols), as a function of the number of dodecyl chains, $n$, for the multi-tailed catanionic ( $n_{1}=2-4$, compounds $\left.1-3\right)$ and multi-charged $\left(n_{2}=4-8\right.$, compounds 4-6) 4-(1-pentylheptyl)benzenesulfonates.

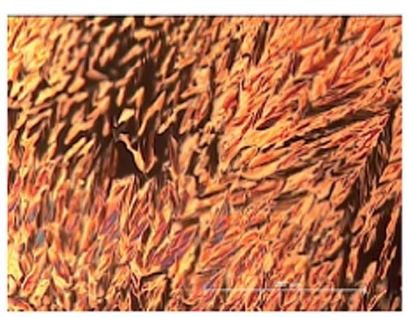

a

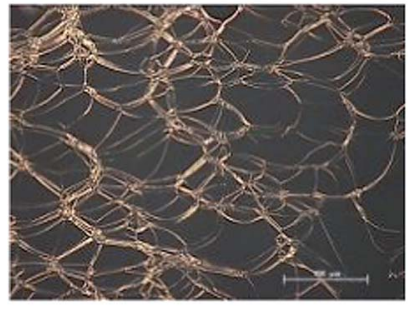

c



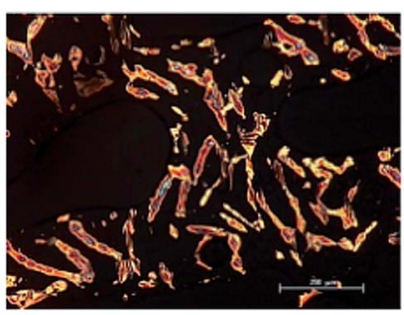

b

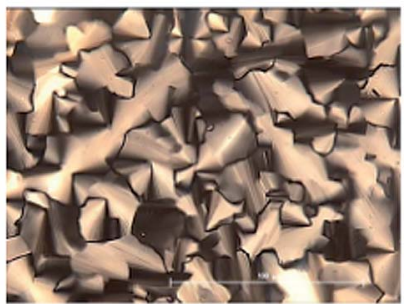

d

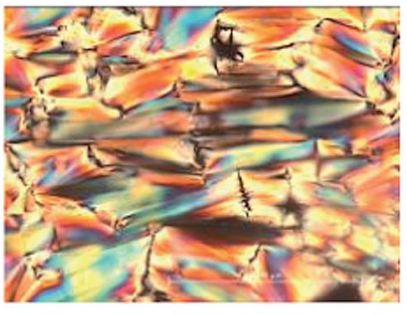

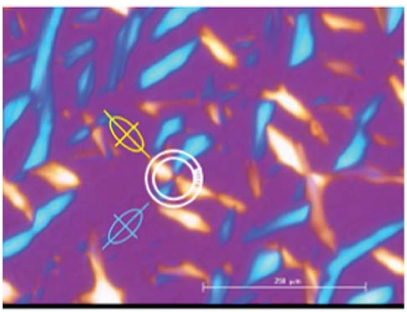
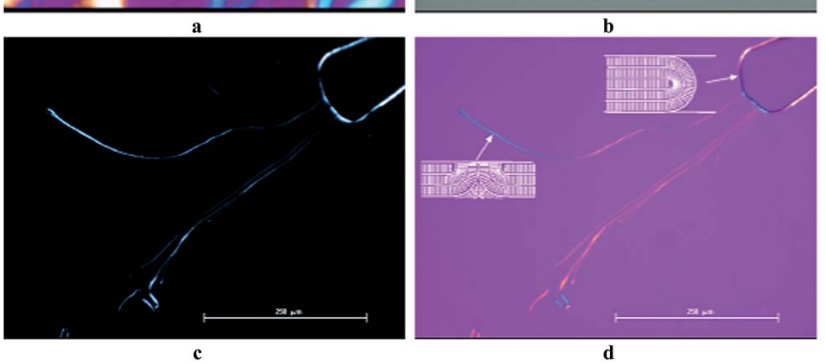

with the maximum temperatures for three-chained catanionic (compound 2). While compound 2 has melting and crystallization temperatures higher than $\mathrm{DDAB},{ }^{47}$ both compounds 2 and 3 exhibit much lower transition temperatures than their bromide analogues. ${ }^{46,48}$ The melting temperatures, $T_{\mathrm{m}_{2}}$, of 4-(1-pentylheptyl)benzenesulfonates (4-6) show the following nonlinear dependence on the total number of alkyl chains in the molecule, $n_{2}$ (Fig. 1):

$$
T_{\mathrm{m}_{2}} / \mathrm{K}=8.3 n_{2}^{2}-72.6 n_{2}+608.1
$$

Fig. 4 Additional optical micrographs. (a) Compound 4 using a fullwave retarder ( $\lambda$-plate) in the SmA phase at $190^{\circ} \mathrm{C}$ after cooling at $10 \mathrm{~K}$ $\mathrm{min}^{-1}$ from the isotropic liquid. More than half than area has homeotropic alignment (layers parallel to the substrate, pink), with numerous conics. In the central area one circular layer is shown schematically in white, with several radial rod-like molecules. Orientation of the projection of the indicatrix is also shown for two of the quadrants, where the long (high-index) axis is parallel to the axis of the rod-like molecules. $(b-d)$ Three micrographs of the compound 5 cooled from the isotropic liquid $\left(170{ }^{\circ} \mathrm{C}\right)$ to smectic phase $\left(160^{\circ} \mathrm{C}\right)$ at $5 \mathrm{~K} \mathrm{~min}^{-1}$. The pictures were taken consecutively from the same area: (b) without polarizers, (c) with crossed polarizers, and (d) with polarizers and $\lambda$-plate. An air bubble is seen at top right, and a few surface disclination lines across the otherwise homeotropic sample. Many such lines are also seen in Fig. 4c. The defects in smectic layers are schematically depicted in the crosssection in (d). The bar corresponds to $250 \mu \mathrm{m}(a-d)$. 
The six-chained catanionic (compound 5) shows the lowest melting temperature. Crystallization temperatures increase with the total number of alkyl chains (or headgroup number) in the catanionic samples that do not decompose. Catanionic compounds 4 and 6 have higher melting temperatures than related bromides, while compound $\mathbf{5}$ melts at a temperature lower than its bromide analogue. Unlike trimer tribromide, ${ }^{\mathbf{4 8}}$ its analogue 4-(1-pentylheptyl)benzenesulfonate does not decompose during melting, while both tetramer tetrabromide ${ }^{48}$ and its analogue 4-(1-pentylheptyl)benzenesulfonate decompose simultaneously under melting. Temperature hysteresis was also observed. It seems to be larger for the simpler molecules in the groups. Moreover, decomposition temperatures of examined catanionic 4-(1-pentylheptyl)benzenesulfonates are higher than those of their bromide analogues. ${ }^{45-48}$ Especially interesting are trends of enthalpy and entropy changes (Fig. 2), which indicate an enhanced disorder of compound 2 (three-chained catanionic), and markedly lower disorder of $\mathbf{5}$ (six-chained catanionic), both during melting and crystallization. As all other examined salts are two-chained $(\mathbf{1})$, four-chained $(3,4)$ or eightchained catanionics (6), it seems that the geometry of molecules based on three and multiples of three alkyl chains makes it difficult for them to pack in ordered arrangements. Such behavior can be explained by the molecular structure of the components itself, and as the consequence, the affinity to make different types of molecular arrangements. In our opinion, disclike shape molecules of sodium 4-(1-pentylheptyl)benzenesulfonate would probably form columns with hexagonal packing properties when not bonded to some other molecules. The layered arrangement of the rod-like catanionics 1 and 3-6 with the total even number of chains is promoted by the quaternary ammonium salts used as cationic components that easily pack their chains, making strong hydrophobic interactions, denser packing, and well-ordered arrangements. On the other hand, the component $\mathrm{DDAB}$ in compound 2 is of similar shape to sodium 4-(1-pentylheptyl)benzenesulfonate, leading to a conclusion that they form a disc-like catanionic molecule 2 , which favorizes less ordered arrangements, leading to stacking into columns.

Fig. 3 shows the characteristic microscopic textures formed upon heating and cooling of the samples. First and second transitions in the DSC (Table 1 ) are characterized as melting and isotropisation for compounds $\mathbf{1 , 5}$ and $\mathbf{6}$, but also as second and third transition for compounds 3 and $\mathbf{4}$. Typical processes can be distinguished upon heating between melting and isotropization, seen as lamellar phase formation, obtained as homeotropic regions with oily streaks (Fig. 3c), and multicolored batonettes (Fig. 3b). The only representative of the second group is compound 2 with fan-like texture before the clearing

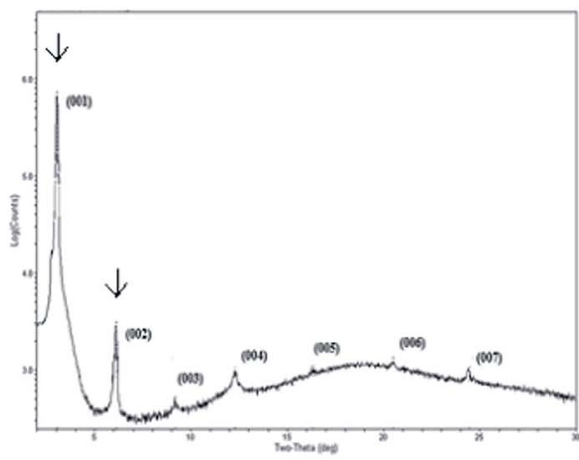

a

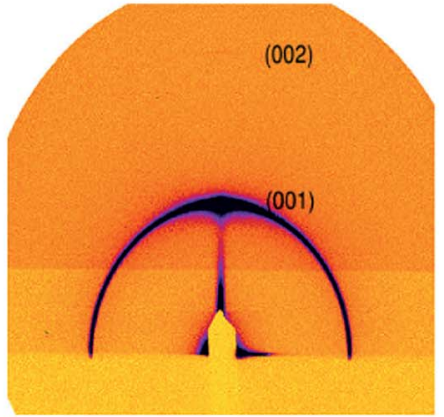

b

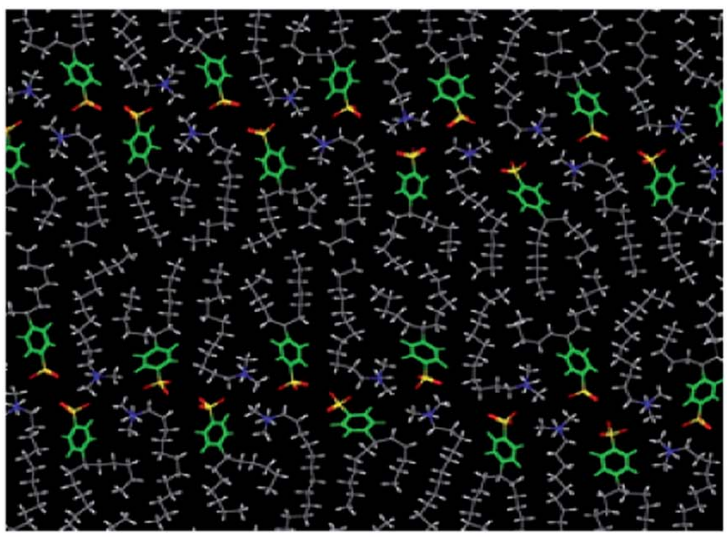

c

Fig. 5 (a) Powder XRD diffractograms of compound 1 recorded at room temperature. (b) GISAXS pattern of a thin film of 1 on the Si substrate recorded at $60^{\circ} \mathrm{C}$. (c) A snapshot of a molecular dynamics simulation of the lamellar phase of 1 at $107^{\circ} \mathrm{C}$, stick model. Benzene rings are colored green for clarity. 
point during heating (Fig. 3a) and before crystallization in the cooling cycle. The optical textures of solid phases 1-5 on cooling are similar, but may point to different types of mesophases. Focal-conic (Fig. 3f), slightly striated (Fig. 3e) point to SmA, or crystal; while broken fan texture (Fig. 3d) may point to the columnar LC. All the LC phase transitions in compounds 1-5 are reversible (enantiotropic). However, reversibility in compound 6 could not be ascertained because the compound decomposes on reaching the isotropic liquid.

Optical textures were also investigated using the full-wave retarder ( $\lambda$-plate). Fig. 4a shows the examples of compound 4. The alignment is mainly homeotropic (optic axis normal to the glass substrate) but with numerous conical defects in which the alignment is planar or nearly planar (optic axis parallel to the substrate). A circular layer is depicted, with radially oriented molecules. The slow axis corresponds to the molecular axis. Fig. $4 \mathrm{~b}-\mathrm{d}$ show one and the same area of compound 5 (b) without polarizers, (c) with crossed polarizers, and (d) with the addition of the $\lambda$-plate. The figures explain the appearance of "oily streaks" in Fig. 3c. "Oily streaks" (Grandjean texture) are common in cholesteric, but less common in smectics. ${ }^{49}$ While at top right we see an air bubble, there are no bubbles elsewhere in the picture. Nevertheless, there are several bright lines visible under polarizers (Fig. 4c). The use of the retarder shows that in both cases the molecules are normal to the lines. The situation, on the molecular scale, is schematically drawn in Fig. $4 \mathrm{~d}$, both at the edge of the bubble and the surface disclination lines which give rise to the "oily streaks". That the black texture between crossed polarizers is indeed homeotropic is also confirmed by a simple shearing experiment. As the cover slip is sheared or pressed the texture brightens up. After the shear the brightness decays within a few seconds. A short video of this experiment made in the real time scale, using compound $\mathbf{5}$ and named "Video, Sample 5 sheared at 175 deg.wmv", is included in the ESI. $\dagger$ Fig. 5a shows a powder X-ray diffractogram of compound 1 at room temperature. Interlayer spacings and Bragg peak intensities at ambient and several other temperatures for the smectic compounds are listed in Table 2. In the diffraction patterns of all samples, there is a diffuse peak near $0.45 \mathrm{~nm}$, characteristic of disordered hydrocarbon chains. Fig. $5 \mathrm{~b}$ shows a grazing-incidence small-angle scattering pattern of compound $\mathbf{1}$ on the Si substrate (horizontal). The strong fundamental (001) Bragg peak is strongly arced, but has a maximum on the meridian confirming the preference for homeotropic alignment. This is consistent with the predominantly black polarized optical microscopy textures between glass surfaces. A weak second order (002) peak is also seen on the meridian (arrow).

Unlike its bromide analogue, DTAB, that shows only solidsolid phase transition to melting of the alkyl chains, without the formation of a thermotropic LC phase, ${ }^{45}$ or crystalline $p$-(trimethylammonio)benzenesulfonate, ${ }^{50}$ compound 1 shows thermotropic mesomorphism. However, the crystals of DTAB have a layer structure stacked along the $c$-axis $(c=2.155 \mathrm{~nm})$, and show tail-to-head packing ${ }^{51}$ i.e. chains lay parallel to one another and headgroups are oriented in the opposite direction. In the crystal of its complex with $p$-phenylphenol, the guest molecules are
Table 2 Interplanar spacings, $d / \mathrm{nm}$, Miller indices, $h k l$, and relative intensities, $I_{\text {rel, }}$ of smectic catanionic 4-(1pentylheptyl)benzenesulfonates at different temperatures

\begin{tabular}{|c|c|c|c|c|}
\hline Compound & $T / \mathrm{K}$ & $d / \mathrm{nm}$ & $h k l$ & $I_{\text {rel }}$ \\
\hline \multirow[t]{10}{*}{1} & \multirow[t]{7}{*}{293} & 2.88 & 001 & 100 \\
\hline & & 1.44 & 002 & 36 \\
\hline & & 0.96 & 003 & 7 \\
\hline & & 0.72 & 004 & 9 \\
\hline & & 0.58 & 005 & 2 \\
\hline & & 0.48 & 006 & 3 \\
\hline & & 0.41 & 007 & 6 \\
\hline & \multirow[t]{3}{*}{400} & 2.86 & 001 & 100 \\
\hline & & 0.95 & 003 & 13 \\
\hline & & 0.48 & 006 & 25 \\
\hline \multirow[t]{8}{*}{3} & \multirow[t]{3}{*}{243} & 2.50 & 001 & 100 \\
\hline & & 0.83 & 003 & 27 \\
\hline & & 0.50 & 005 & 35 \\
\hline & \multirow[t]{3}{*}{253} & 2.76 & 001 & 100 \\
\hline & & 0.92 & 003 & 30 \\
\hline & & 0.46 & 006 & 7 \\
\hline & \multirow[t]{2}{*}{285} & 2.79 & 001 & 100 \\
\hline & & Poorly & & \\
\hline \multirow[t]{7}{*}{4} & \multirow[t]{4}{*}{298} & 2.68 & 001 & 100 \\
\hline & & 1.34 & 002 & 25 \\
\hline & & 0.89 & 003 & 13 \\
\hline & & 0.67 & 004 & 8 \\
\hline & \multirow[t]{3}{*}{468} & 2.97 & 001 & 100 \\
\hline & & 0.99 & 003 & 7 \\
\hline & & 0.50 & 006 & 16 \\
\hline \multirow[t]{6}{*}{5} & \multirow[t]{3}{*}{298} & 2.85 & 001 & 100 \\
\hline & & 1.42 & 002 & 17 \\
\hline & & 0.71 & 004 & 15 \\
\hline & \multirow{3}{*}{443} & 2.61 & 001 & 100 \\
\hline & & 0.87 & 003 & 19 \\
\hline & & 0.44 & 006 & 26 \\
\hline \multirow[t]{3}{*}{6} & \multirow[t]{3}{*}{298} & 2.89 & 001 & 100 \\
\hline & & 1.44 & 002 & 29 \\
\hline & & 0.72 & 004 & 14 \\
\hline
\end{tabular}

included between the DTAB molecules, forming a layer-like structure, again stacked along the $c$-axis that is $c=2.741 \mathrm{~nm} .{ }^{51}$ The insertion of 4-(1-pentylheptyl)benzenesulfonate (compound

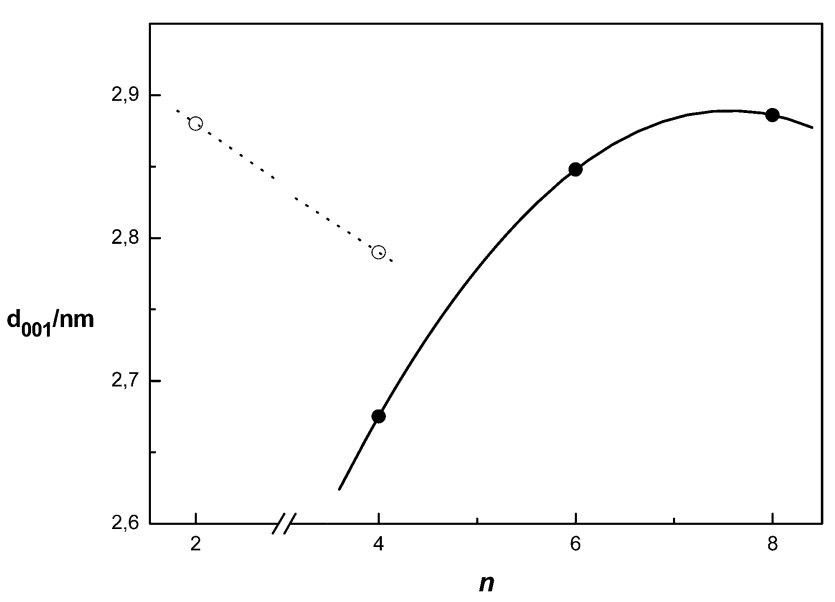

Fig. 6 Values of interlamellar distances as a function of the number of dodecyl chains, $n$, for compounds 1 and $3(O)$; and compounds 4-6 (O) at room temperature. 
1) causes a totally different arrangement. Compound $\mathbf{1}$ is characterized as SmA between RT and $127{ }^{\circ} \mathrm{C}$ (Table 2, Fig. 5). Annealing molecular dynamics simulation of the lamellar phase at $107{ }^{\circ} \mathrm{C}$ (Fig. 5b and c) points to clearly separated hydrophilic layers of polar heads and hydrophobic layers of alkyl chains. Molecular packing can be described as head-to-head with intercalated cationic moieties.
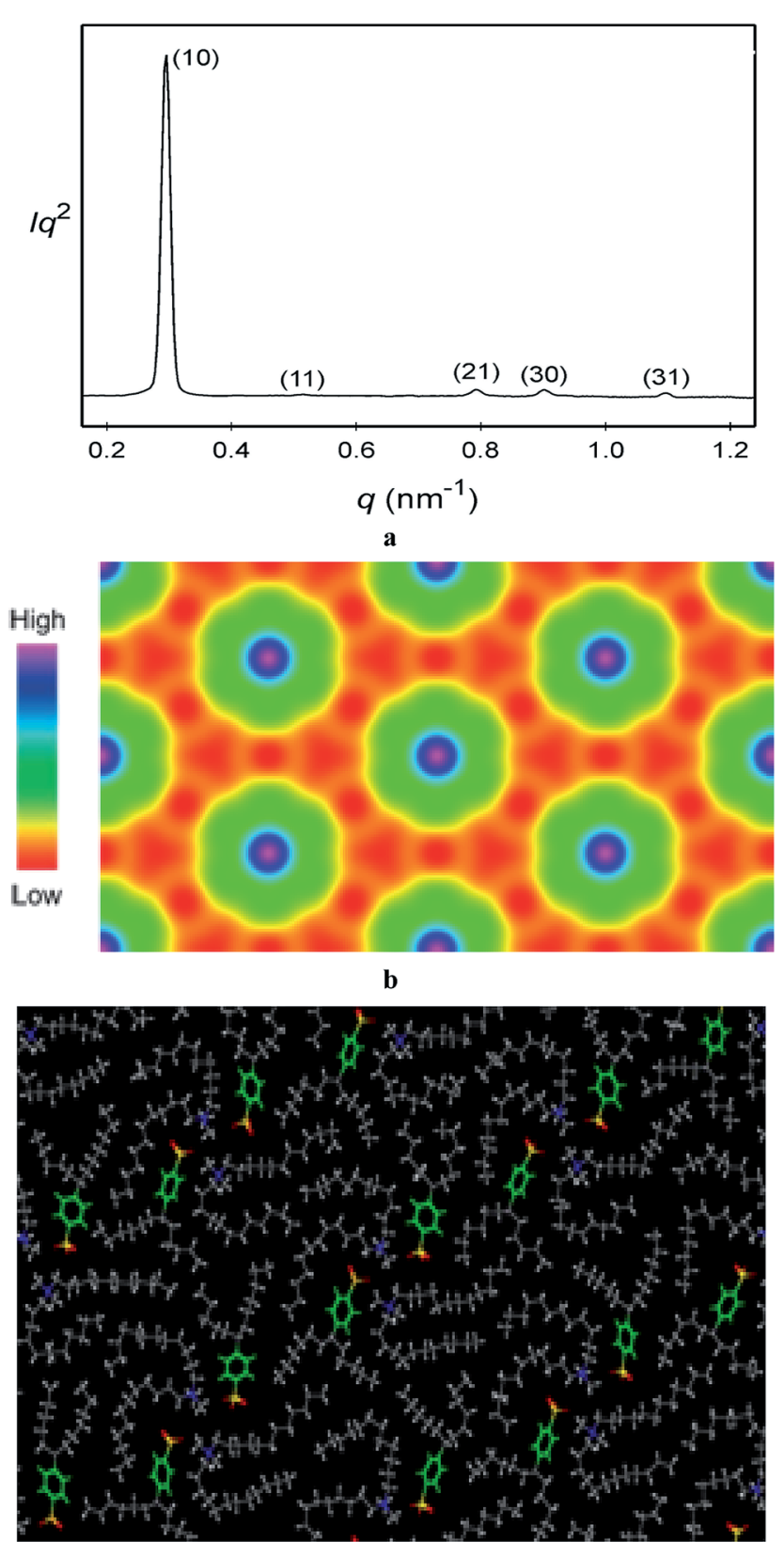

Fig. 7 (a) Small-angle diffraction pattern of compound 2 taken at room temperature. (b) Electron density map, reconstructed using all five observed reflections (purple-blue: high electron density core = sulfate; red: low electron density continuum = alkyl). (c) Snapshot of a molecular dynamics simulation with stick. The cell thickness ("c-dimension") is chosen as $0.45 \mathrm{~nm}$ (one molecular layer). The periodic boundary simulation box contains four unit cells.
Diffractograms of the catanionic samples 3-6 also display sharp peaks with ratio of spacings (Table 2) characteristic for layer arrangement. Their quaternary ammonium bromide analogues also exhibit thermotropic mesomorphism and polymorphism of Sm type. ${ }^{47,48}$ For sample 3 at $12{ }^{\circ} \mathrm{C}$ only one sharp peak at low angle is seen, characteristic for SmA with a low MacMillan (positional) order parameter. As can be seen from Table 2 and Fig. 6 the values of interlamellar distances increase with temperature, and with the degree of oligomerization. However, their decrease is noticed by the introduction of a fourth dodecyl chain (compound 3) in comparison with compound 1, which possesses only two dodecyl chains (Fig. 6). The comparison of interlamellar distances of dodecylammonium-4-(1-pentylheptyl)benzenesulfonate ${ }^{22}$ and those of compound 1 indicates an increase of lamellar thickness above $27 \%$ by the introduction of three methyl groups. In contrast to compounds with varying chain lengths, ${ }^{4}$ compounds of different architectures but the same chain length cause only minor changes of lamellar thickness, indicating their similar layer structure and lamellar packing mode.

Didodecyldimethylammonium bromide component, DDAB, features a crystalline phase at RT, and complex polymorphism at higher temperatures. ${ }^{46}$ There are two LC phases below the isotropic liquid on heating and a third phase appears on cooling. DDAB exhibits highly ordered layered LC structures. The high-temperature phase that appears below the isotropic phase is a SmB phase. However, this double-chained cationic surfactant forms a hexagonal crystal structure with 4-(1-pentylheptyl) benzenesulfonate (compound 2), as also does the doublechained anionic surfactant Aerosol-OT in its alkylammonium catanionic salts. ${ }^{\mathbf{1 4}}$ Experimental $d$-spacings of the observed SAXS reflections at RT stand in the ratio of spacings $1: \sqrt{ } 3: \sqrt{ } 7: \sqrt{ } 9: \sqrt{ } 14$ (Fig. 7a and Table 3 ). The electron density map and the molecular dynamics simulations (Fig. 7b and c) show hexagonal columnar LC arrangement with two ion pairs in each column cross-section, quadrupole in the middle, making $a_{\text {hex }}=2.45 \mathrm{~nm}$. The already mentioned fan-like textures (Fig. 3a) on heating and broken fan-shaped textures (Fig. 3d) on cooling are consistent with a columnar mesophase.

In our opinion, the effect of alkyl chain number in a molecule plays an important role in liquid crystalline structure formation, but its shape greatly affects the molecular structure and as a consequence different phase behavior. The ascending

Table 3 Experimental and calculated $d$-spacings of the observed SAXS reflections of the hexagonal phase of compound 2 at room temperature. All intensities values are Lorentz corrected with correction for multiplicity. The phase can be described as hexagonal columnar, but a relatively sharp peak at $0.47 \mathrm{~nm}$ indicates a relatively high order of the alkyl chains

\begin{tabular}{lllr}
\hline$h k$ & $d_{\text {obs }} / \mathrm{nm}$ & $d_{\text {cal }} / \mathrm{nm}$ & \multicolumn{1}{c}{$I_{\text {rel }}$} \\
\hline 10 & 2.13 & 2.13 & 100.0 \\
11 & 1.22 & 1.23 & 0.3 \\
21 & 0.80 & 0.80 & 1.2 \\
30 & 0.71 & 0.71 & 2.3 \\
31 & 0.58 & 0.59 & 0.9
\end{tabular}


number of polar headgroups in catanionics or individual cationic and anionic surfactants provides a higher number of chains and promotes liquid crystallinity in this great interplay of various possibilities. The linking together of thermodynamics, interaction free energies and geometry results in a general framework which permits extension to more complicated self-assembly problems.

\section{Conclusions}

Thermal and thermotropic behaviors as well as structural properties of new catanionic 4-(1-pentylheptyl)benzenesulfonates were examined using thermogravimetry, thermal polarizing microscopy, differential scanning calorimetry, $\mathrm{X}$ ray diffraction and molecular simulation. New catanionic complexes are $1: 1$ adducts of high purity, and are divided into two groups: 4-(1-pentylheptyl)benzenesulfonates with an increasing number of alkyl chains attached to the cationic headgroup (compounds 1-3) and the ones with an increasing number of ammonium headgroups (compounds 4-6). The compounds studied here are complexes of high thermal stability and rich thermal behavior seen through complex polymorphism and thermotropic mesomorphism. Temperatures of melting for both groups of catanionic compounds show nonlinear dependence on the total number of alkyl chains in the molecule; the melting point decreases with an increasing number of alkyl chains, and increases with an increasing number of ammonium headgroups. Introduction of the third dodecyl chain in the cationic part lowers profoundly the melting point, and prevents the formation of liquid crystallinity. As a result, the compound is isotropic already at RT. The fourth ammonium headgroup in the cationic part reduces markedly the thermal stability of the compound.

Molecular dynamics simulation of the lamellar phase of compound 1 points to clearly separated hydrophilic layers of polar heads and hydrophobic layers of alkyl chains, favoring head-to-head packing of antiparallel molecules. With the exception of compound 2 , all other examined compounds are smectic, both at room and higher temperatures. The introduction of the second alkyl chain, making didodecyldimethylammonium-4-(1-pentylheptyl)benzenesulfonate causes the compound to adopt a hexagonal columnar LC structure instead of the usual smectic. There are two ion pairs in a column cross-section. The examination of the catanionic benzenesulfonate systems provides new clues to help develop strategies for the synthesis of new materials with targeted applicable properties.

\section{Acknowledgements}

This work has received support from the Ministry of Education, Science and Sport of the Republic of Croatia (Project no. 0980982915-2949). Authors would like to thank Dr sc. Ellen J. Wachtel, Weizmann Institute of Science, Jerusalem, Israel, for preliminary temperature X-ray diffraction measurements of the samples.

\section{References}

1 P. Jokela, B. Joensson and A. Khan, J. Phys. Chem., 1987, 91, 3291-3298.

2 E. Marques, A. Khan, M. da Graca Miguel and B. Lindman, J. Phys. Chem., 1993, 97, 4729-4736.

3 N. Filipović-Vinceković, I. Pucić, S. Popović, V. Tomašić and

Đ. Težak, J. Colloid Interface Sci., 1997, 188, 396-403.

4 V. Tomasic, S. Popovic and N. Filipovic-Vincekovic, J. Colloid Interface Sci., 1999, 215, 280-289.

5 A. Khan and E. F. Marques, Curr. Opin. Colloid Interface Sci., 1999, 4, 402-410.

6 E. W. Kaler, K. L. Herrington, A. K. Murthy and J. A. N. Zasadzinski, J. Phys. Chem., 1992, 96, 6698-6707.

7 P. K. Yuet, Langmuir, 1996, 12, 3802-3818.

8 Q. Zhang, M. Tian, Y. Han, C. Wu, Z. Li and Y. Wang, J. Colloid Interface Sci., 2011, 362, 406-414.

9 B. A. Schubert, E. W. Kaler and N. J. Wagner, Langmuir, 2003, 19, 4079-4089.

10 M. Bhat and V. G. Gaikar, Langmuir, 2000, 16, 15801592.

11 Z. Ren, Y. Luo and D. Shi, Colloids Surf. Physicochem. Eng. Asp., 2013, 428, 18-24.

12 V. G. Gaikar, K. V. Padalkar and V. K. Aswal, J. Mol. Liq., 2008, 138, 155-167.

13 C. Richards, G. J. T. Tiddy and S. Casey, Langmuir, 2007, 23, 467-474.

14 G. Ungar, V. Tomasić, F. Xie and X.-b. Zeng, Langmuir, 2009, 25, 11067-11072.

15 N. Filipovic-Vincekovic and V. Tomasic, in Thermal Behavior of Dispersed Systems, ed. N. Garti, CRC Press, 2000, pp. 451476.

16 N. Filipović-Vinceković and V. Tomasic, in Encyclopedia of Surface and Colloid Science: Por-Z, ed. A. T. Hubbard, Marcel Dekker, 2002.

17 D. Tsiourvas, C. M. Paleos and A. Skoulios, Macromolecules, 1999, 32, 8059-8065.

18 F. Mathevet, P. Masson, J.-F. Nicoud and A. Skoulios, J. Am. Chem. Soc., 2005, 127, 9053-9061.

19 B. F. B. Silva and E. F. Marques, J. Colloid Interface Sci., 2005, 290, 267-274.

20 Y. Matsunaga and T. Tsujimura, Mol. Cryst. Liq. Cryst., 1991, 200, 103-108.

21 M. Ito, Y. Matsunaga, H. Matsuzaki and S. Shimojima, Bull. Chem. Soc. Jpn., 1989, 62, 3919-3922.

22 T. Mihelj and V. Tomašić, J. Surfactants Deterg., 2014, 17(2), 1-13.

23 V. Tomašić, S. Popović, L. Tušek-Božić, I. Pucić and N. Filipović-Vinceković, Ber. Buns. Phys. Chem., 1997, 101, 1942-1948.

24 T. Mihelj and V. Tomašić, J. Dispersion Sci. Technol., 2014, 35(4), 581-592.

25 P. K. Yuet, Langmuir, 1996, 12, 3802-3818.

26 V. Tomasic, L. Tusek-Bozic, A. Visnjevac, B. Kojic-Prodic and N. Filipovic-Vincekovic, J. Colloid InterfaceSci., 2000, 227, 427-436. 
27 J.-L. Chai, J.-R. Zhao, Y.-H. Gao, X.-D. Yang and C.-J. Wu, Colloids Surf. Physicochem. Eng. Asp., 2007, 302, 31-35.

28 Z. Nan, X. Chen, Q. Yang, X. Wang, Z. Shi and W. Hou, J. Colloid Interface Sci., 2008, 325, 331-336.

29 H. Wang, K. Huang, C. Huang, S. Liu, Y. Ren and X. Huang, J. Power Sources, 2011, 196, 5645-5650.

30 X. T. Sun and L. Xiang, Cryst. Res. Technol., 2008, 43, 479482.

31 B. Pei, H. Yao, W. Zhang and Z. Yang, J. Power Sources, 2012, 220, 317-323.

32 Q. Yang, Q. Lu, Z. Liu, S. Liu, G. Chen, H. Duan, D. Song, J. Wang and J. Liu, Anal. Chim. Acta, 2009, 632, 115-121.

33 O. Matarredona, H. Rhoads, Z. Li, J. H. Harwell, L. Balzano and D. E. Resasco, J. Phys. Chem. B, 2003, 107, 13357-13367.

34 Z. Chen, S. Qian, J. Chen, X. Chen, L. Zheng and J. Liu, Anal. Bioanal. Chem., 2012, 404, 1673-1679.

35 P. Feng, C. Z. Huang and Y. F. Li, Anal. Bioanal. Chem., 2003, 376, 868-872.

36 Z. Chen, Y. Peng, J. Chen and L. Zhu, J. Pharm. Biomed. Anal., 2008, 48, 946-950.

37 J. Beltrán-Heredia, J. Sánchez-Martín and G. Frutos-Blanco, Sep. Purif. Technol., 2009, 67, 295-303.

38 S. R. Taffarel and J. Rubio, Miner. Eng., 2010, 23, 771779.

39 J. Beltrán-Heredia, J. Sánchez-Martín and C. SoleraHernández, Chem. Eng. J., 2009, 153, 56-61.
40 J.-G. Ma, B. J. Boyd and C. J. Drummond, J. Surf. Colloids, 2006, 22, 8646-8654.

41 Y. Jie, Z. Yu, L. Zongshi, Q. Weihong and C. Lvbo, Energy Sources, 2005, 27, 1013-1018.

42 G. K. H. Shimizu, G. D. Enright, C. I. Ratcliffe, K. F. Preston, J. L. Reid and J. A. Ripmeester, Chem. Commun., 1999, 14851486.

43 X.-Y. Wang, R. Justice and S. C. Sevov, Inorg. Chem., 2007, 46, 4626-4631.

44 O. Danylyuk, B. B. Ghera, A. N. Lazar, A. W. Coleman and K. Suwinska, J. Mol. Struct., 2008, 891, 443-449.

45 K. Iwamoto, Y. Ohnuki, K. Sawada and M. Senō, Mol. Cryst. Liq. Cryst., 1981, 73, 95-103.

46 M. Godlewska, S. Wróbel, B. Borzecka-Prokop, M. Michalec and P. Dynarowicz, Mol. Cryst. Liq. Cryst. Sci. Technol. Sect. Mol. Cryst. Liq. Cryst., 1997, 300, 113-126.

47 T. Mihelj, Z. Štefanić and V. Tomašić, J. Therm. Anal. Calorim., 2012, 108, 1261-1272.

48 D. Jurašin, A. Pustak, I. Habuš, I. Šmit and N. FilipovićVinceković, Langmuir, 2011, 27, 14118-14130.

49 J. M. Pochan and D. G. Marsh, J. Chem. Phys., 1972, 57, 51545156.

50 J. A. R. P. Sarma and J. D. Dunitz, Acta Crystallogr., Sect. B: Struct. Sci., 1990, 46, 784-794.

51 S. Kamitori, V. Sumimoto, K. Vongbupnimit, K. Noguchi and K. Okuyama, Mol. Cryst. Liq. Cryst., 1997, 300, 31-43. 